\title{
Reflexiones psicológicas y epistemológicas acerca de la verdad y la mentira*
}

Verdad y mentira, dos realidades de la vida cotidiana, son analizadas en este articulo, en el cual se considera la naturaleza de ellas en las interacciones interpersonales, así como en la imagen que cada cual desea presentar ante los demás o en la actividad de determinadas profesiones (la diplomacia, por ejemplo). Además de la perspectiva cotidiana de la verdad y de la mentira, la autora presenta consideraciones de naturaleza epistemológica y otras de carácter práctico-estratégicas que las personas emplean a fin de concretizar sus objetivos.

verdad / mentira / epistemología

\section{Psychological and epistemological reflections on truth and lies}

In this essay, truth and lie, two everyday realities are analyzed in their nature whence interacting interpersonally, as well as the image each of us tries to portrait to others, or in the context of specific professions such as diplomacy. Besides the daily perspective shown on truth and lies, the essay discusses the epistemological nature and practical strategies which individuals utilize to fulfill their final objectives. 


\section{LA MENTIRA EN LA VIDA COTIDIANA}

En muchas ocasiones hemos mentido sin experimentar la menor inquietud y mucho menos sentimientos de culpa o remordimientos, y sin ser blanco de críticas por parte de la sociedad.

Por el contrario, si en una reunión le dijéramos a una dama que la encontramos muy fea en lugar de hacer referencia a sus supuestos atractivos, seríamos calificados no solo como personas sin el más elemental tacto, groseros y poco educados. Si declináramos una invitación que no nos interesa sin recurrir a algún tipo de pretexto, expresándole más bien a la persona que nos invita nuestro desinterés, nos ganaríamos la crítica de los circunstantes, dado que habríamos hecho pasar un momento muy desagradable a la persona que pensó en invitarnos. En todos estos casos se trata, como resulta claro, de evitar situaciones desagradables y mantener formas civilizadas. Las buenas maneras, la cortesía y el tacto nos deben llevar a decir alguna cosa que no es verdad.

Todas estas mentiras constituyen formas y reglas de interrelación sancionadas socialmente, no solo toleradas sino inclusive estimuladas, y cuyo incumplimiento nos costará el reproche de la desconsideración, la rudeza o la falta de formas. Se trata, viéndolo bien, de mentiras estandarizadas: pertenecen al código general de conducta de una sociedad que de manera prescriptiva señala que uno debe comportarse de modo tal que se evite problemas y que el trato personal sea fluido, en la medida en que se toma distancia del egoísmo propio y se tolera en el otro debilidades como la vanidad, la conciencia del estatus y el narcisismo.

Albert Vrij, de la Portsmouth University, afirma haber establecido estadísticamente que por lo menos dos veces al día utilizamos mentiras de cortesía, tanto en la vida privada como en la pública, en la familia o en el ámbito profesional (Knill, 2007). La mejor respuesta a la pregunta de por qué se ha llegado tarde a una reunión es la referencia a embotellamientos de tráfico o a algún accidente que determinó el bloqueo de la calle por la cual transitábamos; así como decir que se estaba a punto de responder una carta cuando se nos pregunta por qué hasta ahora no lo habíamos hecho, o señalar que el jefe se encuentra en una reunión cuando se nos pregunta si es posible hablar con él, o que se está con prisa cuando alguien nos solicita una oportunidad para conversar.

Cantidad y magnitud de las falsedades varían al interior de nuestra sociedad. Hay quienes, debido a su función, están casi predestinados al uso de mentiras y eufemismos: la clase política, pero también los importantes líderes de las finanzas y de la economía, en resumen, las personalidades directivas de un país. Al estilo de liderazgo de políticos corresponde en tiempos como estos, de amenazantes crisis económicas, financieras o bancarias, o en épocas en 
que los conflictos comienzan a asumir una configuración peligrosa, el evitar la exposición de la verdad cruda, tal cual ella es, sino más bien el darle un tono rosado o hacerla menos dura, a fin de no desbocar el nerviosismo de la población y hacer que devenga en histerismo.

Frecuentemente estas conductas son interpretadas como una burla a la población o una ofensiva subvaloración de su inteligencia al tratarla como un atado de tontos o limitados mentales, pues esta reconoce muy bien la naturaleza de estas mentiras. Es un hecho, empero, que la presentación brutal de algo podría no ser siempre "digerible" psicológicamente y, por el contrario, ser la causa de un peligroso shock. A través de la información que se da poco a poco, "por cucharadas", la verdad puede finalmente tornarse aceptable. Esto es una ley que la sociología del comportamiento suele destacar. ${ }^{1}$

Cuando los políticos, en tiempos de elecciones, elevan hasta el cielo sus propias capacidades y sus méritos al par que disminuyen o ridiculizan los de sus contrincantes, todos perciben la táctica que está detrás de este proceder, lo cual tiene el efecto de minimizar la mentira: no es que esta desaparezca sino, más bien, pasa a ser socialmente tolerada, y en el peor de los casos será criticada como un estilo político no muy bueno o elegante.

1 Lo mismo vale para alcanzar propósitos y favores, los cuales deben ser elevados sucesivamente a fin de lograr su éxito.
Una muestra de cuán difícil les resulta a los políticos mismos el presentar mentiras como verdades es el hecho de que el expresidente norteamericano George W. Bush tuvo que ser entrenado durante semanas para no mostrar, o al menos controlar en las frecuentes transmisiones televisivas durante la campaña electoral, las señales naturales que suelen emitir el rostro, el cuerpo y la voz cuando se dicen mentiras; por ejemplo, evitar la mirada del otro, elevar los ojos, estrechar los brazos, rascarse nerviosamente detrás de la oreja, elevar el tono de voz, hablar y respirar rápidamente (Ekman, 2011).

En tales circunstancias, las tomas televisivas muy cercanas suelen ser rigurosamente prohibidas, ya que pueden dar a conocer los mínimos cambios que se producen en la musculatura del rostro, o la dilatación de la pupila, señales que, de otro lado, suelen ser constatadas a través del así llamado detector de mentiras.

Un segundo grupo profesional que frecuentemente emplea medias verdades o falsedades lo conforma el servicio diplomático. Formalidades de cortesía, medias verdades, vaguedades, eufemismos, afirmaciones polisemánticas, así como evitaciones, frases crípticas, exageraciones o minimizaciones son moneda de uso diario en el ejercicio de la diplomacia. El trato con interlocutores provenientes de naciones con otra historia y cultura demanda una actuación cuidadosa y meditada a fin de evi- 
tar herir las sensibilidades y los estados de ánimo de estos. La norma aquí es el trato respetuoso y atento, el mismo que es también prescrito en situaciones difíciles que pueden ocasionar alteraciones anímicas en las personas, así como para presentar verdades desagradables, es decir - para decirlo a través de una expresión de uso muy frecuente en el habla castellana- "dorar la píldora" hasta donde esto sea posible.

En dos palabras, la diplomacia ha elevado la mentira social a la categoría de arte. Tanto es así que se habla del estilo diplomático.

Mientras este estilo frecuentemente se caracteriza por un diestro empleo de minimizaciones, otro, muy diferente, recurre con frecuencia a la exageración colorida y cercana al desborde. Nos referimos al lenguaje de los marineros cuando hablan de sus aventuras en medio del océano y en países exóticos. Grupo conformado por gente que permanece largos periodos en alta mar y que pueden llegar a lugares ignotos para el común de las personas, los marineros son proclives a la exageración que transforma un simple pez en un animal de proporciones semejantes a las de Moby Dick, la mítica ballena de Melville, y a una pequeña ola en una impresionante tormenta. Lo mismo puede decirse de las aventuras amorosas de las cuales presumen. En el lenguaje de estas personas es claramente reconocible la estrategia de rodearse de un nimbo misterioso y casi heroico, razón por la cual la mentira pierde su carácter negativo, pues si sus relatos prescindieran de ellas, probablemente serían muy aburridos y nada atractivos. Por ese motivo, exageraciones y desmesuras suelen ser esperadas y son casi legitimadas.

No solo al interior de la sociedad de nuestros días podemos constatar grandes diferencias en el empleo de mentiras sociales. También se las observa en el curso de la historia. Es un tema de discusión si las costumbres que describimos en el marco de este trabajo como mentiras sociales o sencilla y llanamente como cortesía, se desarrollaron con el paso de la rudeza característica de los nobles feudales al progresivo refinamiento de la nobleza cortesana hacia la fase final de la Edad Media, tal como Norbert Elias lo propone en su historia de la cultura (Elias, 1969). La cultura caballeresca en modo alguno fue solo violenta; también estaba regida por la mesura y el autocontrol.

Sí es correcto, sin embargo, que la cultura de la corte de los señores absolutos del siglo XVI y XVII, que ha dado lugar a conceptos como "cortés" y "cortesano", ejerció una gran influencia y contribuyó al origen de las modernas formas de trato y de interrelación personales. Los monarcas absolutos vivían en medio de una espesa red de adulaciones, eufemismos y mentiras practicadas por sus cortesanos, de modo tal que necesitaban a los bufones, "encargados" de decirles sus verdades, pero 
presentadas como burlas, afirmaciones hilarantes o errores cometidos en aparentes descuidos que eran, a su vez, las puertas de escape que hacían posible que el monarca rechazara lo dicho calificándolos como simples locuras.

El hábito de la cortesía con todas sus formas (en muchos casos cercanas al barroquismo) está lejos de haber desaparecido en la actualidad, pero se ha vuelto más sencillo, menos elaborado y ceremonioso que, por ejemplo, lo frecuente aún en el siglo XIX. Esto se puede reconocer en el trato y en las formas de saludo. Lo ocurrido en los años sesenta con la nivelación de las diferencias sociales, expresada -entre otras conductas- por la cancelación de la distinción entre el "Usted" y el "Tú", ha sido a su vez superado, dando lugar al inicio de una nueva búsqueda de formas y órdenes convencionales. A esa búsqueda corresponden la objetividad y la cercanía a la realidad. Junto a la tendencia a la denuncia, al sinceramiento y la revelación está también la tendencia a la vergüenza y la ocultación.

Si bien diariamente nos movemos en un mar de mentiras, lo cierto es que no nos ahogamos ni nos asfixiamos en medio de él sino, por el contrario, nos movemos con soltura y hasta elegancia gracias al proceso de socialización, pues las medias verdades, las mentiras sociales, las afirmaciones que contienen falsedades forman parte de lo convencional, son aprendibles y reconocibles y se nos estimula a usarlas e imitarlas, sin que por ello afecten nuestra confianza en la convivencia social.

Diferente es la atmósfera en la que nos movemos cuando nos encontramos con sociedades y culturas que tienen otros estándares de cortesía y de mentiras, que nos son desconocidos y que, por ello, no podemos identificar con claridad. Esto hace que surja un abismo de desconfianza que nos lleva, como mínimo, a un comportamiento particularmente cauteloso o a inhibirnos. La medida de las mentiras sociales -llamadas cortesía - supera el umbral que podemos tolerar.

Para ser justos, en el análisis de este fenómeno debemos investigar en primer lugar y de manera precisa la verdad extrema y la mentira como concordancia (o ausencia de ella) de una imagen o afirmación con la realidad. En base a la problemática epistemológica de la fundamentación y finalmente de la imposibilidad de demostración de la verdad y de la mentira, surge aquí lo que denominamos una zona gris, ocupada por intereses que no son los epistemológicos sino de índole práctico-estratégica, táctica, que corresponden al plano social y a cuyos fines sirven.

\section{VERDAD Y MENTIRA DESDE EL PUNTO DE VISTA EPISTEMOLÓGICO}

La verdad es definida en la tradición europea por medio de la fórmula tomista de la adaequatio intellectus rei o adaequatio rei et intellectus, es decir 
la concordancia del conocimiento con la realidad, en tanto que la falsedad y la mentira, por el contrario, se definen como la ausencia de tal concordancia (Tomás de Aquino).

Desde una perspectiva dualista se trata de la concordancia o la ausencia de ella de una imagen (Vorstellung) o de una afirmación articulada del sujeto con el mundo objetivo. Esto es conocido como la teoría de la correspondencia, una de las más conocidas teorías acerca de la verdad que atraviesa, a pesar de las numerosas críticas que se le han formulado, toda la tradición occidental y se la encuentra en la actualidad como base de las teorías analíticas.

La constelación expuesta hace que la teoría sea atacable desde tres frentes: el del objeto así como el del sujeto, pero también desde la posición de mediación. Pues si la corrección o la falsedad del conocimiento en relación con el objeto deben ser controladas, se requiere para ello de una instancia, de un tertium comparationis, que sea extramental y extramundana y que solo puede ser entendido, consecuentemente, como un ser sobrenatural, divino, que se convierte en el garante de tal control. Pero esta es, como resulta evidente, una hipótesis metafísica.

Para evitar esto se ha intentado averiguar si en el sujeto hay un don que tiene una directa relación con el mundo objetivo, que es absolutamente cierto y que está por encima de toda duda, sobre cuya base se pueda for- mular una afirmación. El argumento es el siguiente: para demostrar una frase como "en el árbol hay un pájaro" se debe solo mirar, emplear los ojos y otros órganos perceptuales. El argumento no considera, sin embargo, que también las concepciones y percepciones están referidas a la constatación de diferencias y que esa constatación no puede suceder sin preestructuraciones o subestructuraciones. También la observación y la percepción están conceptualmente preeestructuradas e impregnadas por una teoría y por ello dependen del estado anímico y de las cogniciones del sujeto.

Las dificultades de la relación de una imagen inicial/reproducción entre el mundo y el conocimiento se pueden estudiar lo más sencillamente posible en la teoría del lenguaje como relación de correspondencia (Abbildtheorie) de Wittgenstein, en su Tractatus lógicophilosophicus (1922). Si se interpreta la correspondencia entre el mundo objetivo y el conocimiento del sujeto como una relación de imagen o de representación, entonces debe haber entre la imagen esencial y la reproducción tanto semejanzas como diferencias. Si hubiera solo semejanzas, entonces ambas serían lo mismo; si hubiera solo diferencias entonces no serían comparables.

Debe por tanto haber un conjunto de concordancias; en la terminología de Wittgenstein (1922) "la forma de la reproducción", y un conjunto de dife- 
rencias, esto es "la forma de la presentación". De acuerdo con Wittgenstein la estructura lógica debe ser el mínimo que une a la imagen con la reproducción. De acuerdo con esta tesis los componentes de una frase deben concordar en esencia con los componentes de la realidad, aun cuando haya una cantidad de palabras tales como adverbios, conjunciones y proposiciones como "y", "pero", o "si-entonces" que no tienen ninguna relación con la realidad. Para hacer plausible el carácter de reproducción prefiere Wittgenstein ejemplos de relaciones de dos objetos como, por ejemplo, "A pelea con B" o "Romeo ama a Julieta", en una notación formal escritos como $a R b$. En esta relación el objeto reproducido es claramente reconocible. Como la palabra "pelear" está entre el nombre de A y B y la palabra "ama" entre Romeo y Julieta, entonces permanece en la realidad imitada a través de este modelo la relación de pelea y de amor entre dos personas. La frase, en su configuración, puede por tanto ser considerada como la representación de lo que ocurre.

Las cosas, sin embargo, se complejizan en predicados de un solo objeto, como "Carlos es rubio". El carácter de reproducción ya no es más plausible aquí, y mucho menos en casos en los cuales se intentan reproducir en la realidad conceptos como "todos", "porque", "si-entonces". Ellos no pueden ser explicados sobre la base de una homogeneidad estructural (Patzig, 1970).
Si se considera, además, que no solo hay lenguajes de la familia lingüística indogermánica sino también otros que están estructurados de una manera muy diferente, que solo operan con sustantivos como la lengua japonesa o sobre todo con verbos como el idioma de los esquimales, la teoría del carácter de la reproducción no puede mantenerse. Ese fue el motivo por el cual Wittgenstein en su fase tardía evolucionó hacia una teoría lúdica del lenguaje (Sprachspieltheorie). También esta vía de argumentación va en la dirección de que el lenguaje no reproduce una realidad dada, sino que más bien estructura la realidad. En la consecuencia está un enfoque trascendental-lingüístico en analogía con el enfoque trascendentalfilosófico de Kant, de acuerdo con el cual las condiciones del conocimiento o las características del lenguaje forman la realidad.

Dado que fracasa el intento de establecer una prueba de validez para una afirmación de acuerdo con la teoría de la correspondencia, surge como salida solo una retirada total o al mundo objetivo o al dominio subjetivo. Una teoría de la primera índole es la que propone Martin Heidegger en su teoría óntica de la verdad presentada en Sein und Zeit (Heidegger, 1947, 2001), con la cual él se vincula a la antigüedad griega. Remitiéndose a la palabra griega $\alpha \lambda \eta \theta \varepsilon \iota \alpha$, que se compone de la raíz $\lambda \alpha \theta-, \lambda \eta \theta$ - con el sentido de 
"ocultar" y "olvidar" y el privativo $\alpha$ y tiene el sentido de Unverborgenheit (no soledad) y Unverstellheit (no disimulo), propone la verdad como la autorrevelación del objeto, como mostrar el objeto desde sí y, consecuentemente, la falsedad como ocultación y disimulo. Por cierto, Heidegger evita a través de la inmediatez de la autopresentación del objeto, que no requiere ninguna otra prueba más que ella misma, la problemática de la mediatez de la relación; sin embargo, está ausente en esta interpretación todo criterio comunicable objetivo, intersubjetivo de corrección o falsedad de un conocimiento. La verdad se reduce aquí a la simple y pura convicción subjetiva, la cual-como se sabe-puede asumir muy diversas formas. Para el conocimiento y comprensión requerimos, sin embargo, una comunicación intersubjetiva.

La otra posibilidad consiste en el recurso al sujeto y sus condiciones de conocimiento y estructuras del lenguaje inmanentes. Si el programa de una concordancia de las afirmaciones con el mundo no se puede realizar, porque los objetos del mundo se reducen a una esencia incognoscible y todo lo que nosotros podemos saber tiene el status de una realidad conocida y expresada por nosotros, entonces no queda otra cosa que establecer la verdad y la fal-

2 En la mitología griega los muertos deben cruzar el río Leteo, el río del olvido, para llegar a la otra orilla. sedad en la concordancia o la no-concordancia de una afirmación con todas las otras afirmaciones de un sistema de afirmaciones. Consistencia y coherencia se convierten aquí en criterios decisivos sobre la verdad y la falsedad. Una afirmación debe concordar con todas las otras. La diferencia externa entre afirmación y objeto se reduce así a una diferencia al interior del lenguaje entre una afirmación más pobre en contenido, más abstracta, y otra más rica y más concreta, la cual abarca todo lo que por lo demás se pueda decir sobre el objeto. El mundo no es algo que está más allá del conocimiento, sino un determinado sistema del lenguaje rico en contenido.

El origen de esta teoría de la coherencia es inconfundible: de un lado representa una crítica al empirismo lógico del Círculo de Viena y de la teoría de la correspondencia como en el caso de Otto Neurath (1931); del otro, ella es la formulación de una posición idealista, como en el caso de Brand Blanshard (1939).

Empero, también se han formulado objeciones sobre el particular, especialmente las de Bertrand Russell (1977). Un escritor particularmente dotado de fantasía podría imaginar y construir un mundo regido por leyes y en concordancia con todo lo que nos es conocido, y que, sin embargo, al mismo tiempo no tendría nada que ver con nuestro mundo.

En la ciencia hay frecuentemente hipótesis concurrentes, que cubren todos los hechos conocidos y relevantes pero 
que al mismo tiempo no guardan concordancia entre sí. La decisión por una $\mathrm{u}$ otra propone un criterio no científico, esto es el criterio estético, el cual prefiere la sencillez, la belleza y la elegancia de una teoría.

También en la filosofía es frecuente que dos conceptos que rivalizan entre sí luchen con respecto a la verdad. Por ejemplo, sería posible que toda nuestra vida fuera un largo sueño y que el mundo exterior en estado de conciencia no pudiera reclamar para sí la condición de realidad.

Como no hay ningún criterio para diferenciar entre estado de conciencia y de sueño tampoco hay criterio alguno que permita separar la verdad de la supuesta falsedad.

\section{Verdad y MENTIRA DESDE EL PUNTO DE VISTA PRÁCTICO-ESTRATÉGICO}

Si el resultado de las valoraciones epistemológicas es que no existe ninguna prueba definitiva de la verdad y de la mentira, y que no hay instrumento alguno para la diferenciación entre ambas, entonces se origina un vacío en el cual en lugar de una búsqueda de un conocimiento puramente objetivo surgen intereses y valoraciones de naturaleza práctico-estratégicas. La insistencia en la diferencia entre la verdad y la mentira se integra entonces con el ethos de lo honesto y de lo deshonesto. Estos pueden aparecer en todos los niveles y variedades, comenzando por la honradez, la integridad, la veracidad, hasta el subterfugio, el ocultamiento, el secreteo, la deshonestidad, el engaño, la mentira, la estafa y la infamia. Una afirmación verdadera en estas circunstancias es aquella que expresa mi honesta convicción (u opinión) acerca de algo o de una situación, y una falsedad sería aquella que de manera más o menos consciente se aleja de eso. No hay criterios objetivos para la determinación de esto, solo existen los subjetivos y también estos pueden cambiar en el transcurso de la vida o en base a un análisis particularmente profundo, dando lugar a nuevas formas de valorar la realidad.

Con esto el problema se traslada de un plano epistemológico a otro práctico-estratégico, y ético o moral.

Puesto que la moral tiene que ver con la relación entre una persona con ella misma y con su entorno, sea este sociedad o naturaleza, se trata acá de motivos, propósitos e intereses que mueven a un individuo a elegir una forma determinada del espectro total de posibilidades entre los extremos de la más profunda convicción personal y de la más pronunciada ausencia de convicciones. Los intereses van desde el cuidado y el bienestar de la propia persona, así como del grupo al cual ella pertenece, hasta la agresión dirigida a otros, desde la búsqueda de reconocimiento y motivaciones egolátricas hasta la promoción altruista del grupo. Esto explica las sutiles diferencias que solemos hacer entre una mentira 
social o lo que llamaremos una mentira de cortesía consciente y tolerada convencionalmente (o aún promovida) y una declaración falsa premeditada o un juramento falso, entre un embuste chistoso acompañado de un expresivo pestañeo y una declaración que busca engañarnos, entre un intento con rasgos irónicos de embellecer una situación y un eufemismo con fines estratégicos, o entre una adulación servil y un disimulo bienintencionado.

Las estrategias empleables en las cuales se pueden ordenar las formas intermedias de la verdad y de la mentira son:

a) Estrategias prosociales

1. estrategias de toma de contacto;

2. estrategias de fortalecimiento o de renovación de la solidaridad grupal;

3. estrategias de mantenimiento de la armonía interpersonal y la del grupo; $y$,

4. estrategias de búsqueda personal de rangos, de jerarquía y de subordinación de los otros.

b) Estrategias disociales, agonales

1. estrategias para causar daño a otros con el fin de proteger a la propia persona o el grupo;

2. estrategias de luchas contra el enemigo; $\mathrm{y}$,

3. estrategias de imposición personal y de destrucción del otro (Eibl-Eibesfeldt, 1997).
Esta clasificación puede ampliarse, pero permite ya en esta forma reconocer las dos grandes tendencias que determinan la elección de las formas y medios estratégicos: las tendencias prosociales y las tendencias antisociales.

A las primeras corresponden las numerosas formas de saludo y de expresión de respeto, comenzando por el jovial "hola", pasando por formas de saludo de validez regional (por ejemplo, "Grüss Gott", en Baviera) o los neutrales "buenos días" y "buenas noches", hasta las formas oficiales establecidas por la convención, como por ejemplo los deseos de éxito y suerte, los buenos augurios, las comunicaciones escritas de agradecimiento y las inclinaciones corporales. ${ }^{3}$ Nadie que en la calle le dice a otra persona que pasa a su lado "buenos días" medita sobre esa expresión y le desea de manera genuina un hermoso día; más bien, se trata de una forma de saludo establecida por las normas de buena educación y que no expresa una carga afectiva positiva o negativa determinada. Lo mismo vale para el cortés "muchas gracias" que se expresa por escrito u oralmente, aun cuando uno no se alegre en absoluto por el favor o el obsequio recibidos. También detrás de los deseos de suerte y de éxito para la salud o el logro de alguna meta puede

3 En el Japón las personas se inclinan varias veces según el respeto y la veneración que experimentan por su interlocutor. 
estar oculto el deseo opuesto: en estos casos estamos ante simples fórmulas verbales a ser usadas en las ocasiones en que nos parezcan apropiadas. Su función es servir tan solo al establecimiento, preservación o restablecimiento de una relación social.

Igualmente, corresponde a esto el intento de granjearse la amistad de una persona determinada mediante la exaltación de su belleza, su inteligencia u otras capacidades, todo lo cual no es algo que corresponda a lo que realmente pensamos de ella sino que tiene por finalidad únicamente el promover el inicio de una relación o de una atmósfera agradable en el trato interpersonal. Expresiones de simpatía y de valoración del otro unidas a la disminución de nuestro propio valor favorecen una apertura por parte del interlocutor y pueden contribuir así a la obtención de los propios objetivos. En entrevistas de trabajo o determinadas situaciones ocurre una sincronización real o fingida de los intereses del postulante con los de la empresa y su personal, lo que debe permitir que establezca una base común de propósitos. También aquí mucho de lo que se dice y se actúa es fingido, en modo alguno real, pudiendo en el mejor de los casos ser valorado como una exageración con el propósito de ganarse a la persona o instancia con la cual se sostiene la entrevista.

No menos variadas son las formas y rituales que enfatizan la pertenencia a un grupo o la unidad y armonía de este ante la amenaza de una ruptura o de grietas que minen la solidaridad y el compromiso. Las personas, en estos casos, reiteran su compromiso personal, sin que mediten mucho sobre el particular; se prometen recíproca colaboración y ayuda de una manera convencional y casi mecánica. Se emplea también la táctica de la calificación en blanconegro y en amigo-enemigo como era lo que sucedía en los tiempos de la Guerra Fría entre Occidente y Oriente, con el propósito de fortalecer los vínculos aun cuando se perciba claramente que se trata de exageraciones. Se minimizan, disimulan, perdonan o ignoran muchas cosas en el otro cuando surge la amenaza de la ruptura sin que la minimización, el perdón y la ignorancia correspondan a lo que realmente pensamos y sentimos. Con motivo de las más pequeñas causas la aversión al otro puede volver a manifestarse. Importantes en el trato y las relaciones políticas de los estados entre sí son determinados rituales así como expresiones de interés, respeto y amistad con un fuerte componente demostrativo en caso de peligro de conflictos, estrategias de engaño, reclamos y concesiones por parte del otro. Se trata de simples formalidades y no de propósitos serios, cuya finalidad es mantener las relaciones sociales y los contactos amistosos y no permitir el surgimiento de malos entendidos y agresiones.

Dado que las jerarquías también forman parte del orden social, incluso 
en democracias, la búsqueda de estas y de rangos tiene también un rol muy importante, y consiste en gestos y poses corporales, técnicas de ubicación en el centro de los acontecimientos o de la atención de los demás y en el autoelogio. Si bien es posible reconocer estas técnicas, se las tolera pues las jerarquías también traen ventajas para la comunidad y sirven para la protección de los subordinados.

Mientras las estrategias prosociales emplean las mentiras en sentido positivo, es decir con la finalidad de establecer, conservar o renovar la solidaridad grupal y, por ello, son valoradas positivamente por la sociedad, las estrategias antisociales o antagonistas se proponen debilitar y combatir al enemigo, rival u oponente con la finalidad de proteger a la propia persona o al grupo. Las mentiras empleadas con este fin son valoradas según diferentes puntos de vista, sea desde la perspectiva del amigo o de la del enemigo.

Un rol importante juegan las mentiras en la autodefensa que emplean los delincuentes, cuyos delitos y fechorías han sido descubiertos y que, por ese motivo, intentan disminuir la magnitud de sus actos delincuenciales. En ningún otro contexto se dicen tantas mentiras como en los juzgados, algo que todo juez sabe pero que es tolerado socialmente, hasta cierto punto, sin que ocasione mayores consecuencias penales. Sin embargo, sí surgen tales efectos en casos de perjurio y de difamación de otra persona, así como en estafas y afirmaciones engañosas.

Por el contrario, las mentiras en las entrevistas de trabajo así como los datos incorrectos en el currículum vítae, el ocultamiento de aspectos negativos, las informaciones falsas en lo que concierne a calificaciones, las relaciones con anteriores empleadores, los sueldos percibidos y otras cosas por el estilo, no suelen dar lugar a consecuencias penales (Bundesarbeitsgericht, 1963).

Cuán elástico es nuestro trato con este tipo de asuntos lo demuestran, en una perspectiva histórica, las siempre variables sentencias judiciales. Según estudios de grupos de control los alemanes deben ser los campeones en materia de falsedades: $30 \%$ de datos acerca de la ocupación son falsos, $18 \%$ acerca de las calificaciones, $13 \%$ en torno a los sueldos previamente ganados, $11 \%$ acerca del currículum vítae, $6 \%$ de los referidos a las relaciones con el antiguo empleador; $4 \%$ acerca de las posiciones profesionales alcanzadas, y $1 \%$ acerca de las posiciones de liderazgo logradas (Spreng \& Dietrich, 2006).

Estrategias explícitamente agonistas de lucha contra el enemigo o el rival consisten en expresiones verbales exageradas acerca del valor de la propia persona y del grupo al cual se pertenece y la correspondiente minusvaloración de la persona opuesta y de su grupo, pero también en aparentes fortalezas físicas, psicológicas, intelectuales o económicas, junto a ofensas, 
estrategias de asesinato caracterológico y desmoralización del rival. Llamamos a esto bluffen, pero en grado extremo mobbing. Por parte de los que pertenecen al mismo grupo tales técnicas son bienvenidas y aprobadas, por parte de los contrarios denunciadas y condenadas. Dado que estas estrategias tienen por finalidad engañar o en el mejor de los casos confundir, son claramente reconocibles.

Debe resultar claro que las formas de trato y de comportamiento que hemos clasificado dentro del rubro de mentiras sociales no solo tienen que ver con contenidos verbales articulados sino también con actos verbales, con la solicitud de favores, ruegos, preguntas, deseos, órdenes, amenazas, bendiciones y maldiciones. Para el logro de nuestros objetivos hay diversos niveles y grados, que se determinan según la cercanía o la lejanía, la extensión o la intensidad.

Irenäus Eibl-Eibesfeldt (1997) se refiere a investigaciones llevadas a cabo por Penélope Brown y Stephen Lewinson, que tenían por finalidad explorar las solicitudes corteses entre los tamiles, los tzeltan (México) y los ingleses. En ellas se encontró de manera transcultural una misma estructura de los tres niveles, que reflejaban primero, intimidad; segundo, formalidad; y en tercer lugar, convencionalidad (ritualidad).

En solicitudes corteses o favores en el círculo íntimo se suele hacer referencia a una base común o se la finge en caso necesario. En la elaboración de esta primera relación de cooperación se aplica el plural "nosotros" en vez del "tú" y "yo"; se necesitan palabras afectuosas como "mi corazón" que destacan la valoración que le damos al otro: "Tú eres una magnífica persona, ¿me pueden hacer tal o cual favor?".

La cortesía formal, por el contrario, se caracteriza por la distancia, el respeto y las formas convencionales. Se ponen de manifiesto las maneras en la medida en la cual se emplean formas establecidas por la etiqueta, tales como: “PPodría usted, por favor, ...?, "Tal vez le sería posible...". El tercer ritual de cortesía consiste en que se dice expresamente lo que se desea o se sugiere algo, pero se deja abierta la posibilidad de que el otro acceda a lo sugerido o solicitado de modo tal que el malestar asociado a la negativa de lo solicitado sea mínimo, tanto en el solicitante como en aquel que debe acceder a la solicitud. Un ejemplo es: "Que día tan caluroso el de hoy, sería bueno tomar un refresco"; "hace frío en el cuarto, sería bueno cerrar las ventanas".

Si bien tales niveles de estrategias de cortesía se encuentran como constantes en todas las culturas, existe una gran diferencia entre una y otra. Cada cultura establece una zona gris entre la verdad y la mentira según sus necesidades e intereses sociales. Las formas respectivas son aprendidas en el proceso de socialización a través del tiempo. Cuanto más lejana es una cultura 
de la nuestra tanto más inseguros nos sentiremos en el trato con ella (crossrace-effect). Cuanto más cerca estemos de integrantes de nuestra cultura, tanto más confiables serán ellos (group advantage), cuanto más distantes tanto menos confiables. Las formas entre la verdad y la mentira, sea la cortesía, la vaguedad, la evitación, la exageración, el disimulo, entre otros, están basadas en la sociedad y son formas tácticas de trato que no tienen nada que ver con las formas epistemológicas. En el mejor de los casos las sociedades pueden conceder valor al ethos de la verdad y de la integridad, es decir, de las convicciones personales.

\section{RefERENCIAS}

Blanshard, B. (1939). The nature of thought. Londres: Allen \& Unwin.

Bundesarbeitsgericht 19. Mai 1983 (2 AZR 171/181; BAG 2 AZR 227/92).

Eibl-Eibesfeldt, I. (1997). Die Biologie des menschlichen Verhaltens, Grundriss der Humanethologie. Munich: Piper [1964].

Ekman, P. (2011). Ich weiss, dass $d u$ lügst. Was Gesichter verraten. Reinbeck b. Hamburg: Rowohlt.

Elias, N. (1969). Ueber den Prozess der Zivilisation, Soziogenetische und psychogenetische Untersuchungen (2 vols). Berna-Munich: Francke [1939].
Heidegger, M. (1947). Platons Lehre von der Wahrheit. Berna: Francke.

Heidegger, M. (2001). Sein und Zeit. (18 a ed.) Tubinga: Niemeyer [1927].

Knill, M. (2007). Kommunikation und Lüge. Recuperado el 26 de octubre del 2010, de http://www.rhetorik.ch/ Wahrheit/Wahrheit1.html

Neurath, Otto (1931). Soziologie im Physikalismus. En: Erkenntnis (vol. 2, pp. 393-431).

Patzig, G. (1970). Sprache und Logik. Göttingen: Vandenhoeck und Ruprecht.

Russell, B. (1977). Wahrheit und Falschheit. En: Gunnar Skirbek, Wahrheitstheorien, Eine Auswahl aus den Diskussionen über Wahrheit im 20. Jahrhundert. Frankfurt a. M. (pp. 60-72).

Spreng, N. M., \& Dietrich, S. (2006). Studien- und Karriere-Ratgeber für Juristen. Berlín-Heidelberg-Nueva York: Springer.

Tomás de Aquino. (1986). Von der Wahrheit/De veritate. Edición latínalemán seleccionada, traducida y editada por Albert Zimmermann, Hamburgo, p. 8 (Quaestio I, art. 1), p. 14 (Quaestio I, art . 2).

Wittgenstein, L. (1922). Tractatus logico-philosophicus. Londres: Kegan Paul, Trench, Trubner \& Co. 